\title{
Prediction of ground surface settlement induced by twin shield tunnellings in urban conditions
}

\author{
Thai Ngoc Do* , Truong Duc Nguyen \\ Hanoi University of Mining and Geology, Hanoi, Vietnam
}

ARTICLEINFO ABSTRACT

Articlehistory:

Received 10th Sep. 2020

Revised 20th Jan. 2021

Accepted 12 ${ }^{\text {th }}$ Feb. 2021

\section{Keywords:}

Finite element method, Shield tunneling machines, Surface settlements, Tunnel.
Tunneling in urban areas is growing in response to the increased needs for efficient transportation. Many urban tunnels are constructed in soft ground at shallow depths. Metro tunnels are usually constructed as twinparallel tunnels and their adjacent constructions may lead to surface deformation, affecting the surface environment and the safety of the tunnels. Shield tunnelling is a commonly used as construction technique because it is very effective in reducing ground deformations and thus damage to urban infrastructure. The paper presents a $3 D$ simulation of shield tunneling machines via the finite element code Abaqus and analysis model of ground surface settlements induced by a construction of twinparallel tunnels. The results show that ground surface settlements induced by a construction of the left tunnel causes surface settlements of about $22 \div 24 \mathrm{~mm}$ and after the construction of both tunnels, it will cause ground subsidence has the greatest value of $33 \div 35 \mathrm{~mm}$.

Copyright (C) 2021 Hanoi Universityof Mining and Geology. All rights reserved.

${ }^{*}$ Corresponding author

E-mail: dongocthai@humg.edu.vn

DOI: 10.46326/JMES.2021.62(2).05 


\title{
Nghiên cứu dự báo độ lún mặt đất khi thi công hai đường hầm song song trong đô thị bằng máy khiên đào
}

\author{
Đỗ Ngọc Thái *, Nguyễn Đức Trường \\ Trường Đại học Mỏ - Địa chất, Hà Nội, Việt Nam
}

\begin{abstract}
THÔNG TIN BÀI BÁO

\section{Quátrình:}

Nhận bài 10/9/2020

Sửa xong 20/01/2021

Chap nhận đăng 12/02/2021

Tù̀ khóa:

Độ lún mặt đất,

Đường hầm,

Máy khiên đào,

Phương pháp phần tử hữu hạn.

\section{TÓMTẮT}

Công tác xây dưng đường hầm trong đô thị đang rất phát triển để đáp úng nhu cầu cấp thiết của vấn đề giao thông vận tải. Có nhiều đường hầm trong đô thi được bố trí nằm nông, thi công trong đấtyếu. Các đường hầm tàu điện ngầm trong đô thị thường xây dựng dưới dạng hai đường hầm song song nằm gần nhau, nên khi thi công có thể dẫn đến hiện tượng lún mặt đất, phá hủy các công trình trên mặt đất hoặc ảnh hưởng đến độ ổn định của chính các đường hầm. Máy khiển đào thường được sử dung để thi công đường hầm trong đô thị vì công nghệ thi công có thể giảm thiểu độ lún mặt đất hay giảm thiểu tác đông đến các công trình trên mặt đất. Bài báo sủ dụng phương pháp phần tử hữu han thông qua phần mềm Abaqus-3D đế mô phỏng và phân tích dự báo độ lún mặt đất khi thi công hai đường hầm song song. Kết quả tính toán đối với đường hầm trong điều kiên phân tích, tác động tù̀ công tác thi công đường hầm bên trái gây ra độ lún mặt đất có giá trị lớn nhất là $22 \div 24 \mathrm{~mm}$ và sau khi thi công cả hai đường hầm thì gây ra độ lún mặt đất có giá trị lớn nhất là $33 \div 35 \mathrm{~mm}$.
\end{abstract}

(C) 2021 Trường Đại học Mỏ - Địa chất. Tất cả các quyền được bảo đảm.

\section{Mở đầu}

Hiện nay, ở nước ta nhu cầu xây dựng các đường hầm tàu điện ngầm trong thành phố là rất lớn, các thành phố Hà Nội và Hồ Chí Minh đang bắt đầu xây dựng hệ thống tàu điện ngầm để đáp ứng nhu cầu phát triển kinh tế - xã hội. Thi công đường hầm tàu điện ngầm trong thành phố có những tác động gây dịch chuyển, sụt lún mặt đất làm ảnh hưởng tới độ ổn định của các công trình

"Tác giả liên hệ

E-mail: dongocthai@humg.edu.vn DOI: 10.46326/JMES.2021.62(2).05 trên mặt đất như nhà cửa, cầu, đường, các công trình hạ tầng kỹ thuật khác (Võ Trọng Hùng và Phùng Mạnh Đắc, 2005; Do Ngoc Thai và Protosenya, 2017). Vì vậy công tác dự báo giá trị độ lún mặt đất khi thi công đường hầm tàu điện ngầm trong thành phố đóng vai trò rất quan trọng. Ngày nay, phương pháp thi công bằng máy khiên đào được áp dụng rộng rãi trong xây dựng các đường hầm tàu điện ngầm thành phố. Phương pháp thi công bằng máy khiên đào ngoài việc đảm bảo chất lượng công trình, độ ổn định cho đường hầm còn giảm thiểu được những ảnh hưởng chấn động, dịch chuyển lún mặt đất hay bảo vệ các công trình xung quanh khu vực thi công. Máy 
khiên đào là máy đào hầm cơ giới có nhiều chức năng tập trung thống nhất như: đào tách khối đất đá, che chống bảo vệ, lắp đặt vỏ hầm, vận chuyển đất đá. Máy khiên đào thích hợp cho việc thi công đường hầm qua vùng đất đá mềm yếu, phức tạp có nguy cơ mất ổn định cao, đất đá có khả năng sụt lở ngay vào trong không gian công trình nếu không có kết cấu chống giữ.

Hiện nay, có nhiều phương pháp dự báo độ lún mặt đất, có thể kể đến một số phương pháp chính như: phương pháp phân tích lý thuyết; phương pháp bán thực nghiệm và phương pháp mô hình số. Tuy nhiên, các phương pháp đều đặc biệt chú trọng đến các yếu tố ảnh hưởng đến độ lún mặt đất khi thi công đường hầm như: đặc tính địa kỹ thuật khối đất đá mà đường hầm thi công qua, các thông số kỹ thuật đường hầm và công nghệ thi công đường hầm.

\section{Phương pháp dự báo độ lún mặt đất}

\subsection{Phương pháp dự báo lún mặt đất khi thi công tuyến đường hầm đơn}

Hiện nay có nhiều phương pháp dự báo độ lún bề mặt đất, có thể kể đến một số phương pháp chính như: phân tích lý thuyết; bán thực nghiệm và mô hình số. Phương pháp bán thực nghiệm được các nhà nghiên cứu Peck R.B. (1969) và Schmidt B. (1974) là những người đầu tiên đề xuất bằng cách đo một số điểm tại hiện trường, kết quả thu được là dưới tác động của quá trình thi công đường hầm thì trên mặt đất sẽ hình thành phễu lún (Hình 1).

Khi thi công đường hầm trong môi trường đất đồng nhất, đẳng hướng thì gây ra độ lún trên mặt có giá trị $\left(\mathrm{S}_{\mathrm{v}}\right)$ được xác định theo công thức (1), đường cong lún bề mặt đất được Peck R.B. (1969) giả định có dạng hàm phân phối chuẩn Gauss hay đường cong phân phối chuẩn, với điểm lún cực đại $\left(\mathrm{S}_{\mathrm{v} \cdot \max }\right)$ nằm ngay trên trục thẳng đứng của đường hầm:

$$
\mathrm{S}_{\mathrm{v}}=\mathrm{S}_{\mathrm{v} \cdot \max } \cdot \mathrm{e}^{-\left[\mathrm{x}^{2} /\left(2 \mathrm{i}^{2}\right)\right]}
$$

Trong đó: $\mathrm{S}_{\mathrm{v} \text { max }}$ - giá trị độ lún lớn nhất theo phương thẳng đứng dọc trục đường hầm, $\mathrm{m} ; \mathrm{x}$ khoảng cách từ trục hầm theo phương nằm ngang, $\mathrm{m}$; $\mathrm{i}$ - khoảng cách từ tâm đường hầm đến điểm uốn theo phương nằm ngang, $\mathrm{m}$.

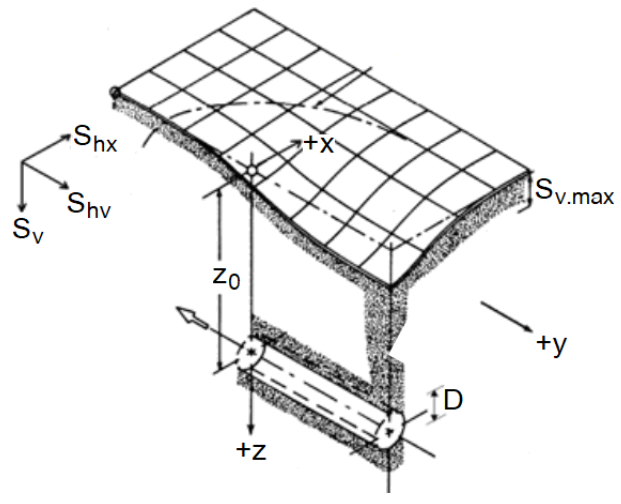

Hình 1. Hình dạng phễu lún trên mặt đất sau khi thi công đường hầm.

Theo O’Reilly và New (1982) giá trị khoảng cách từ tâm đường hầm đến điểm uốn theo phương nằm ngang (i) được xác định theo công thức:

$$
\mathrm{i}=\left(\mathrm{k} \cdot \mathrm{z}_{0}\right)
$$

Trong đó: $\mathrm{k}$ - tham số chiều rộng phễu lún, phụ thuộc vào điều kiện và loại đất mà đường hầm thi công qua, ví dụ đối với cát trong điều kiện nước ngầm thì $\mathrm{k}=0,2 \div 0,3$ và đối với đất sét thì $\mathrm{k}=$ $0,4 \div 0,7 ; z_{0}$ - chiều sâu bố trí đường hầm, $m$.

Thể tích $\left(\mathrm{V}_{\mathrm{s}}\right)$ trên mỗi đơn vị chiều dài đường hầm được xác định theo công thức:

$$
\mathrm{V}_{\mathrm{v}}=\int_{-\infty}^{\infty} \mathrm{S}_{\mathrm{v} \cdot \max } \mathrm{e}^{-\left[\frac{\mathrm{x}^{2}}{2 \mathrm{i}^{2}}\right]}=\sqrt{2 \pi} \cdot \mathrm{i} \cdot \mathrm{S}_{\mathrm{v} \cdot \max }
$$

Lượng mất thể tích đất $\left(\mathrm{V}_{\mathrm{L}}\right)$ là tỷ số giữa thể tích của phễu lún và thể tích đào lý thuyết tính cho một đơn vị chiều dài.

$$
\mathrm{V}_{\mathrm{L}}=\left(\mathrm{V}_{\mathrm{s}} / \mathrm{V}_{\mathrm{o}}\right) \cdot 100 \%
$$

Trong đó: $\mathrm{V}_{\mathrm{o}}$ - thể tích đào lý thuyết, $\mathrm{m}^{3}$.

Lượng mất thể tích là do sự khác biệt về thể tích đào đường hầm và thể tích hoàn thành sau khi lắp đặt vỏ chống. Đất xung quanh đường hầm di chuyển để lấp đầy sự mất thể tích này, cường độ di chuyển lấp thể tích cũng gây ra lượng mất thể tích, giá trị mất thể tích còn phụ thuộc vào phương pháp đào, loại đất đá mà công trình đào qua và sự thận trọng của đơn vị thi công đường hầm. Một phần của lượng hao hụt thể tích đất xung quanh hầm sẽ phát triển lên đến mặt đất và tạo ra phễu lún. Hay nói cách khác, thể tích phễu lún trên mặt đất tương ứng với lượng mất thể tích đất xung quanh đường hầm (Dimmock và Mair, 2008). 
Từ các công thức $(1 \div 3)$ độ lún tại điểm bất kỳ trên mặt đất được xác định theo công thức:

$$
S_{v}=\frac{V_{S}}{\sqrt{2 \pi} \cdot K \cdot z_{0}} \cdot e^{-\left[x^{2} /\left(2 k^{2} \cdot z_{0}^{2}\right)\right]} .
$$

Có rất nhiều công trình nghiên cứu như quan sát thực địa và kiểm tra bằng mô hình số để dự báo các giá trị $S_{v \text { max }}$ và i trong các điều kiện thi công khác nhau. Các giá trị đó phụ thuộc vào đặc tính kỹ thuật đường hầm, điều kiện địa chất khu vực xây dựng đường hầm.

Phương pháp dự báo lún mặt đất khi thi công đường hầm tàu điện ngầm thành phố có thể sử dụng phương pháp giải tích hoặc phương pháp mô hình số. Để dự báo giá trị lún mặt đất gây ra từ công tác thi công một đường hầm cụ thể có thể kết hợp nhiều phương pháp dự báo và so sánh với kết quả đo đạc, quan trắc thực tế của các công trình có điều kiện xây dựng tương tự.

\subsection{Phương pháp dự báo lún mặt đất khi thi công hai đường hầm song song}

Giá trị lún mặt đất gây ra bởi công tác xây dựng đường hầm đơn có thể được dự báo bởi các phương trình của Peck (1969), O'Reilly và New (1982) nhưng khi thi công hai đường hầm song song thì tác động của công tác thi công đường hầm gây ra hiện tượng lún mặt đất phân bố rộng và lớn hơn so với trường hợp một đường hầm đơn duy nhất (Peck, 1969; O'Reilly và New, 1982; Đỗ Ngọc Anh, 2016) như trên Hình 2.

Theo Divall S. và Goodey R.J. (2012), độ lún mặt đất khi thi công hai đường hầm song song được xác định theo công thức:

$$
\mathrm{S}_{\mathrm{v}}=\mathrm{S}\left[\exp \left(-\frac{\mathrm{x}_{\mathrm{A}}^{2}}{2 \mathrm{i}^{2}}\right)+\exp \left(\frac{\left(\mathrm{x}_{\mathrm{A}}-\mathrm{L}\right)^{2}}{2 \mathrm{i}^{2}}\right)\right]_{\max }
$$

Trong đó: L - khoảng cách theo phương ngang giữa 2 tâm đường hầm, m; $\mathrm{x}_{\mathrm{A}}$ - khoảng cách ngang từ tim đường hầm đầu tiên đến điểm cần tính lún, m.

Để dự báo độ lún mặt đất khi thi công hai đường hầm song song bằng máy khiên đào trong điều kiện địa chất tuyến Metro số 3 đoạn Nhổn Ga Hà Nội, Thành phố Hà Nội, trong nghiên cứu này sử dụng phương pháp phần tử hữu hạn thông qua phần mềm chuyên dụng Simulia Abaqus-6.12 dựa trên tiêu chuẩn phá huỷ Mohr-Coulomb, phần mềm cho phép phân tích các quá trình thi công tách bóc đất đá, duy trì áp lực ổn định gương đào, công tác lắp dựng vỏ hầm và công tác phụt vữa lấp đầy khoảng trống giữa bề mặt đất đá và vỏ hầm, đồng thời đưa ra các kết quả giá trị ứng suất và dịch chuyển khối đất đá gây ra bởi công tác thi công đường hầm.

\section{Xây dựng mô hình số}

\subsection{Kích thước mô hình}

Kích thước mô hình có ảnh hưởng đến tốc độ tính toán, độ chính xác của kết quả tính toán, vùng phân tích được lựa chọn có kích thước phương nằm ngang, phương thẳng đứng và phương dọc trục hầm, có giá trị lần lượt là $120 \times 80 \times 100 \mathrm{~m}$. Đường hầm có đường kính $\mathrm{D}=6,3 \mathrm{~m}$, độ sâu đặt đường hầm $\mathrm{H}=20 \mathrm{~m}$, khoảng cách tính từ tâm hai đường hầm $\mathrm{L}=15 \mathrm{~m}$, vỏ hầm là bê tông cốt thép đúc sẵn có các thông số: chiều dày $\mathrm{d}=0,3 \mathrm{~m}$, mô đun đàn hồi $\mathrm{E}_{0}=25 \times 103 \mathrm{Mpa}$, hệ số Poisson $v_{0}=0,2$. Các thông số cơ lý khối đất đá tuyến Metro số 3 đoạn Nhổn - Ga Hà Nội, Thành phố Hà Nội được thể hiện trong Bảng 1.

Bảng 1. Thông số cơ lý khối đất đá sử dụng trong mô hình.

\begin{tabular}{|c|l|c|c|c|c|c|c|c|c|c|}
\hline STT & $\begin{array}{c}\text { Lớp đất } \\
\text { đá }\end{array}$ & $\begin{array}{c}\text { Chiều } \\
\text { dày }\end{array}$ & $\begin{array}{c}\text { Dung } \\
\text { trọng tự } \\
\text { nhiên, } \\
\gamma_{\text {unsat }} \\
\left(\mathrm{kN} / \mathrm{m}^{3}\right)\end{array}$ & $\begin{array}{c}\text { Trọng } \\
\text { lượng } \\
\text { bão hòa, } \\
\gamma_{\text {sat }} \\
\left(\mathrm{kN} / \mathrm{m}^{3}\right)\end{array}$ & $\begin{array}{c}\text { Hệ số } \\
\text { lỗ } \\
\text { rô̂ng, } \\
\mathrm{e}\end{array}$ & $\begin{array}{c}\text { Mô } \\
\text { đun } \\
\text { đàn } \\
\text { hồi, } \mathrm{E} \\
(\mathrm{MPa})\end{array}$ & $\begin{array}{c}\text { Hệ số } \\
\text { Poisson, } \\
\nu\end{array}$ & $\begin{array}{c}\text { Góc } \\
\text { ma } \\
\text { sát } \\
\text { trong, } \\
\varphi\left({ }^{0}\right)\end{array}$ & $\begin{array}{c}\text { Góc } \\
\text { giãn } \\
\text { nở, } \\
\Psi \\
(0)\end{array}$ & $\begin{array}{c}\text { Lực } \\
\text { dính } \\
\text { kết, c } \\
(\mathrm{MPa})\end{array}$ \\
\hline 1 & Sét mềm & 5 & 19 & - & - & 10 & 0,35 & 12 & 0 & 0,25 \\
\hline 2 & Cát & 7 & 19,3 & 20 & 0,2 & 15 & 0,35 & 18 & 0 & 0,30 \\
\hline 3 & Sét & 21 & 20 & 21 & 0,4 & 20 & 0,32 & 30 & 0 & 0,30 \\
\hline 4 & Đá gốc & - & 22 & 22,5 & 0,4 & 80 & 0,3 & 35 & 0 & - \\
\hline
\end{tabular}


Khoảng cách ngang trục giữa hai đường hầm, $\mathrm{m}$

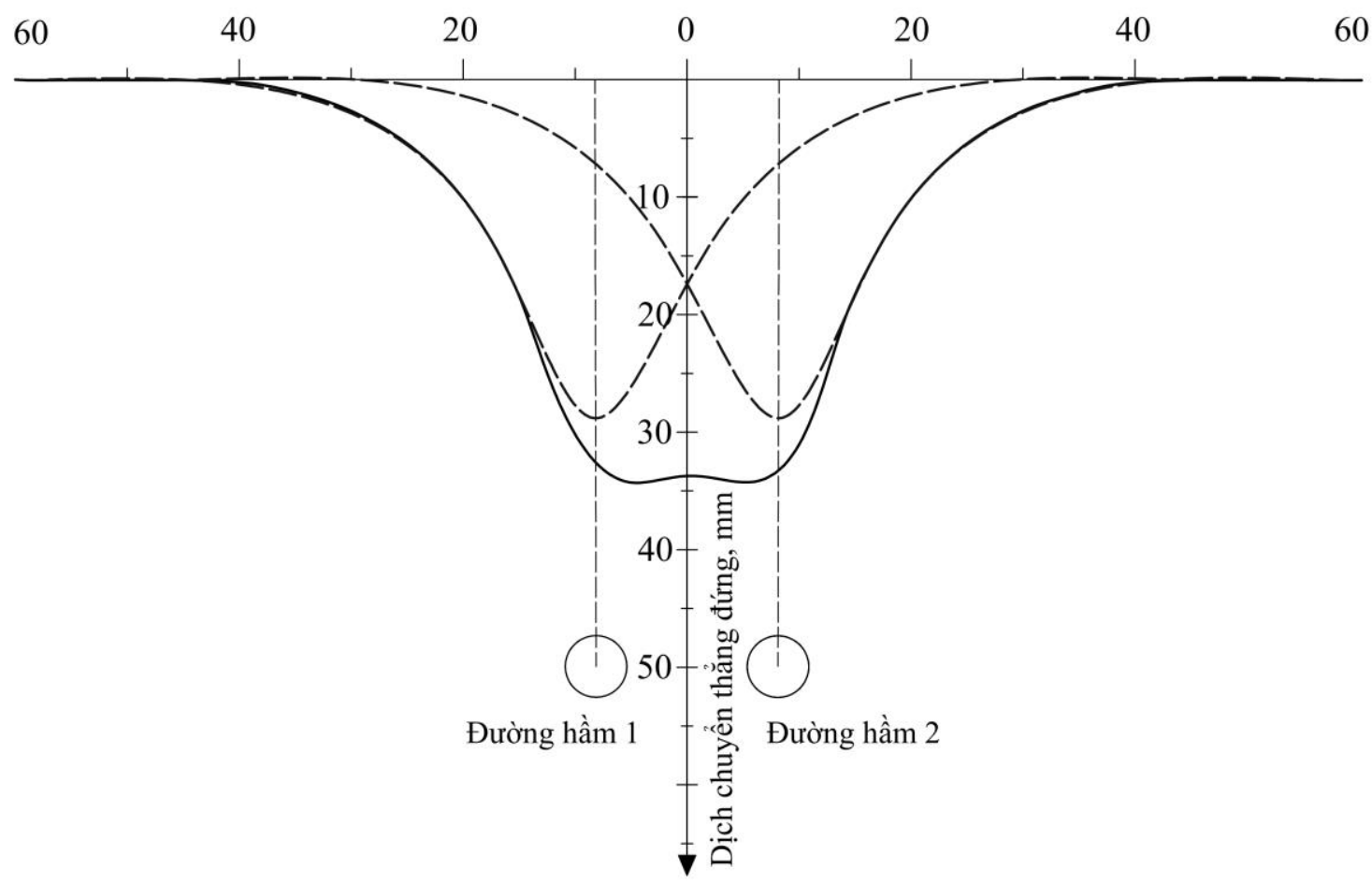

Hình 2. Dự báo dịch chuyển lún mặt đất khi thi công hai đường hầm song song.

(Theo Divall S. và Goodey R.J. 2012).

\section{2. Điều kiện biên}

Biên trái và biên phải của mô hình chọn loại cố định có ứng suất tiếp và chuyển vị ngang tại biên bằng không; ứng suất pháp và chuyển vị thẳng đứng để tự do. Biên đáy của mô hình có chuyển vị ngang, thẳng đứng bằng không; ứng suất tiếp, pháp tuyến để tự do. Biên phía bề mặt để tự do cho phép chuyển vị thẳng đứng và chuyển vị ngang. Điều kiện biên và vị trí các lớp đất đá như trên các Hình $3,4$.

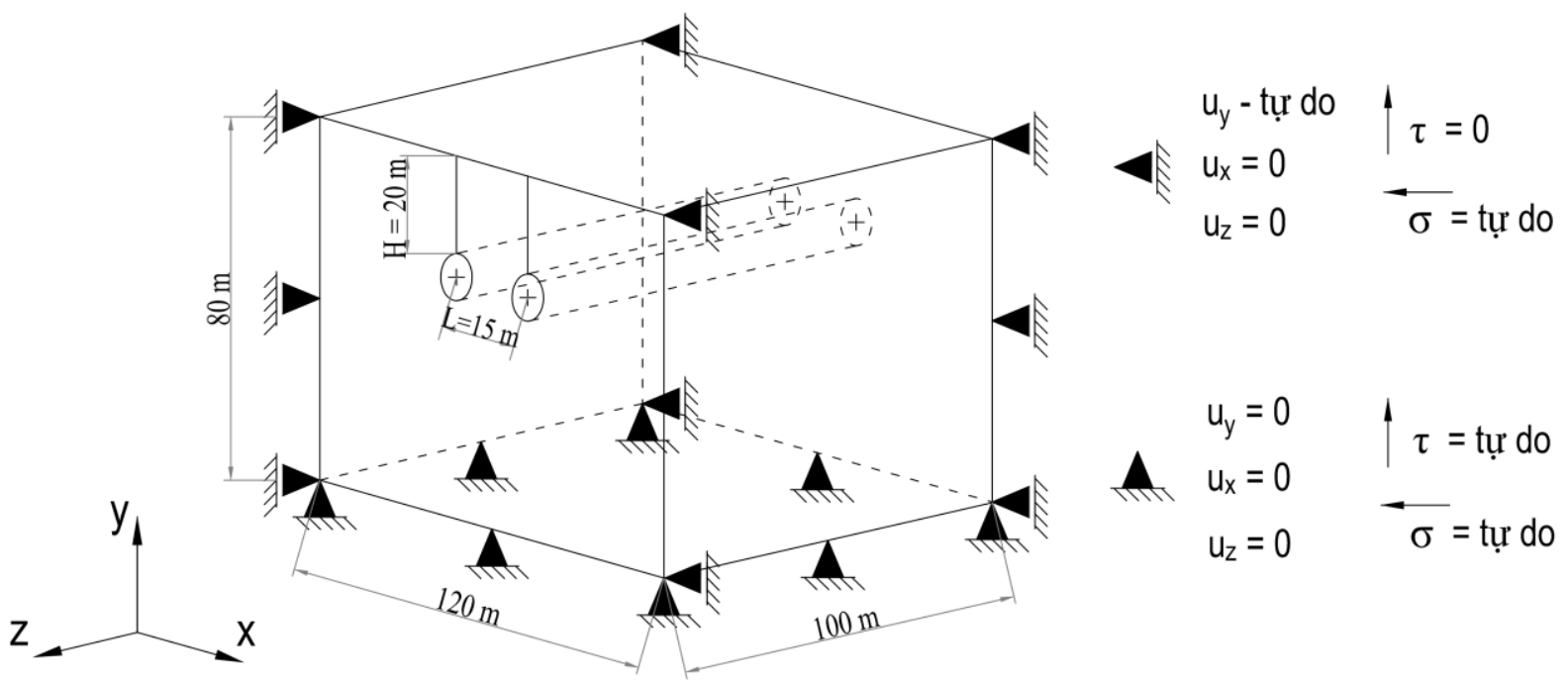

Hình 3. Điều kiện biên bài toán. 


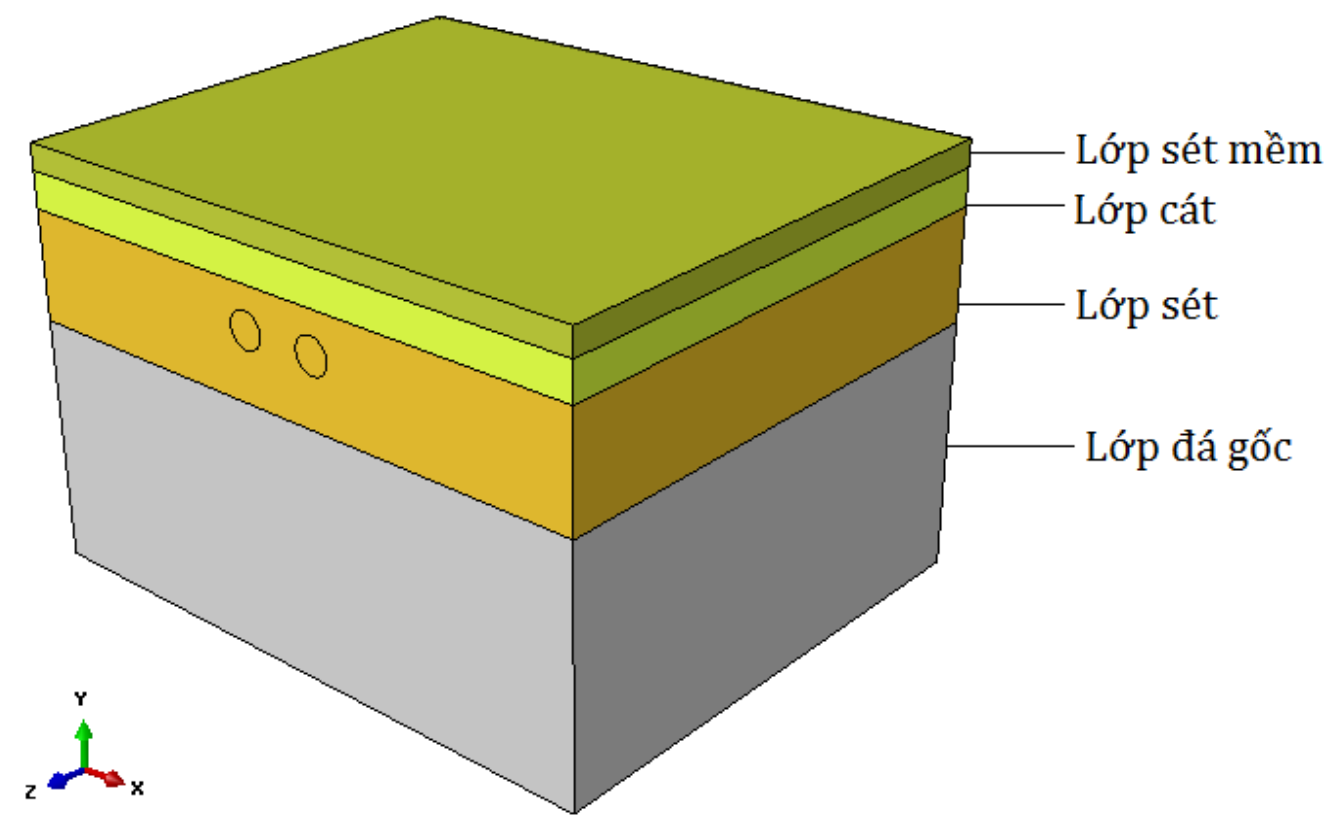

Hình 4. Vị trí các lớp đất đá.

\section{3. Áp lực lên gương đào}

Ở bài toán này, sử dụng áp lực lên gương đào có giá trị phân bố tuyến tính tăng theo độ sâu của gương đào: $\mathrm{F}_{\mathrm{g}}=150 \mathrm{kPa}+\Delta \mathrm{F}$. $\mathrm{H}_{\text {guơng }} \mathrm{kPa}$, trong đó: $\Delta \mathrm{F}$ - giá trị áp lực gương tăng tuyến tính theo độ sâu từ đỉnh hầm đến đáy hầm, $\Delta \mathrm{F}=12 \mathrm{kPa} / \mathrm{m}$;
$\mathrm{H}_{\text {gương }}$ - độ sâu gương đào, tại đỉnh gương: $\mathrm{H}_{\text {guoong }}$ $=0 \mathrm{~m}$, tại đáy gương đào: $\mathrm{H}_{\text {gưong }}=6,3 \mathrm{~m}$. (Hình 5).

\subsection{Kết quả tính toán}

Kết quả mô phỏng lún mặt đất gây ra bởi công tác thi công đường hầm được thể hiện trên Hình 6.

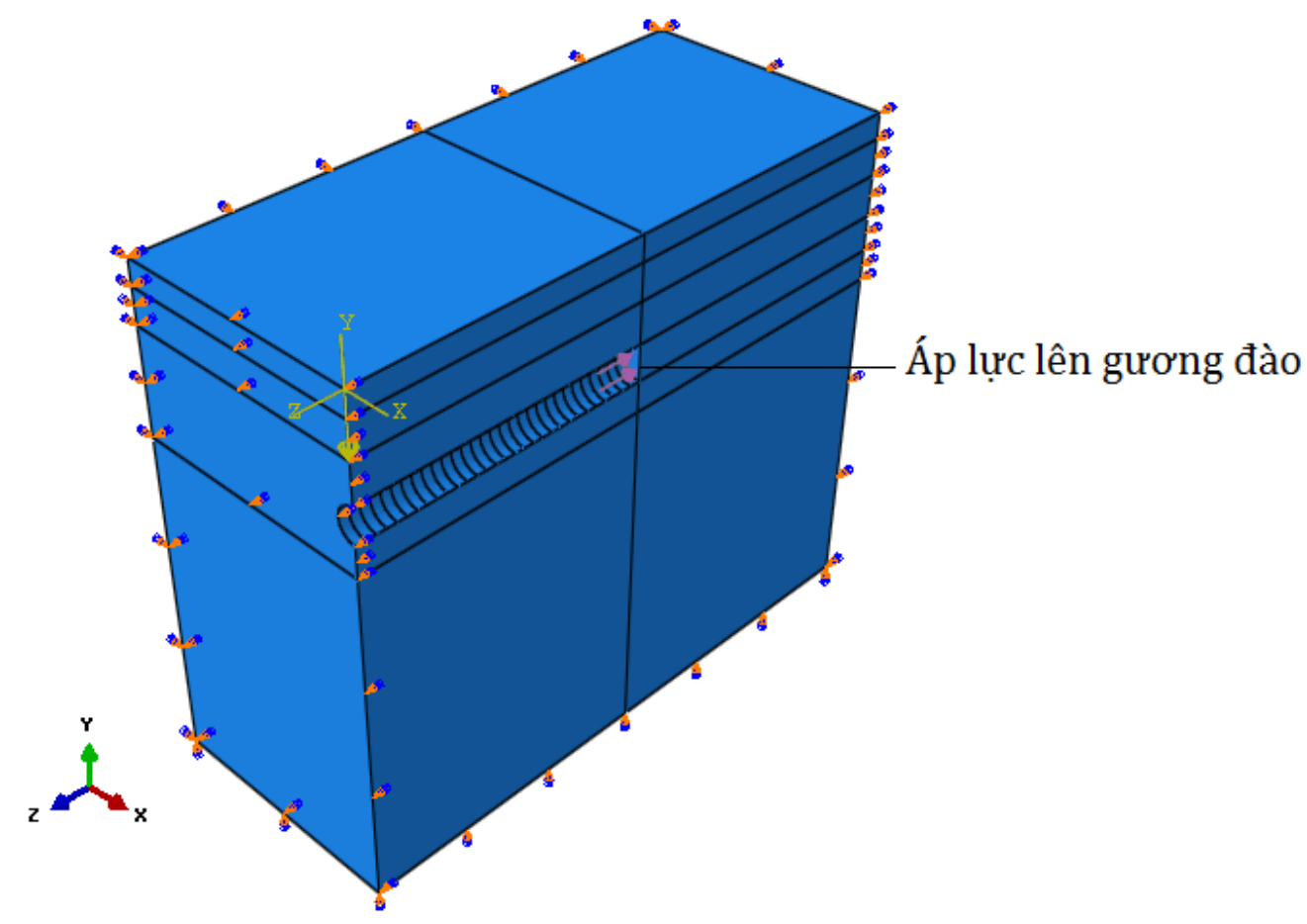

Hình 5. Duy trì áp lực lên gương đào đường hầm. 

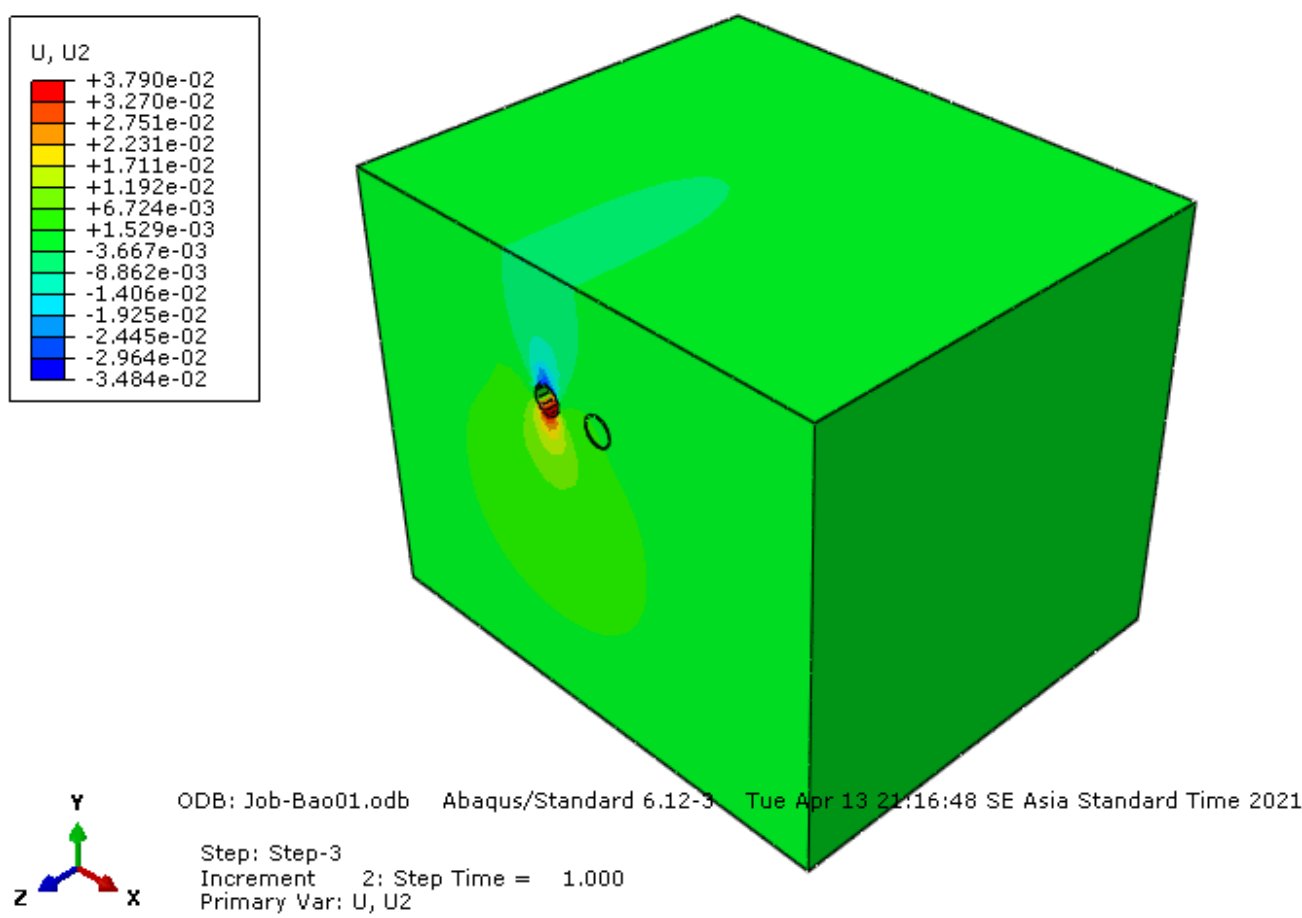

a) Sau khi thi công đường hầm bên trái.

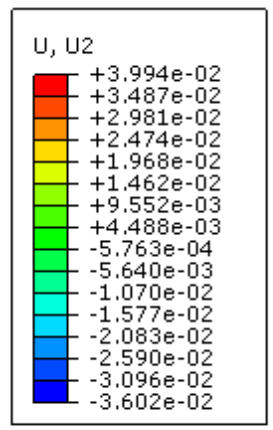

$\begin{array}{ll}\text { Step: Step-3 } & \text { 2: Step Time }=1.000\end{array}$

Primary Var: U, U2

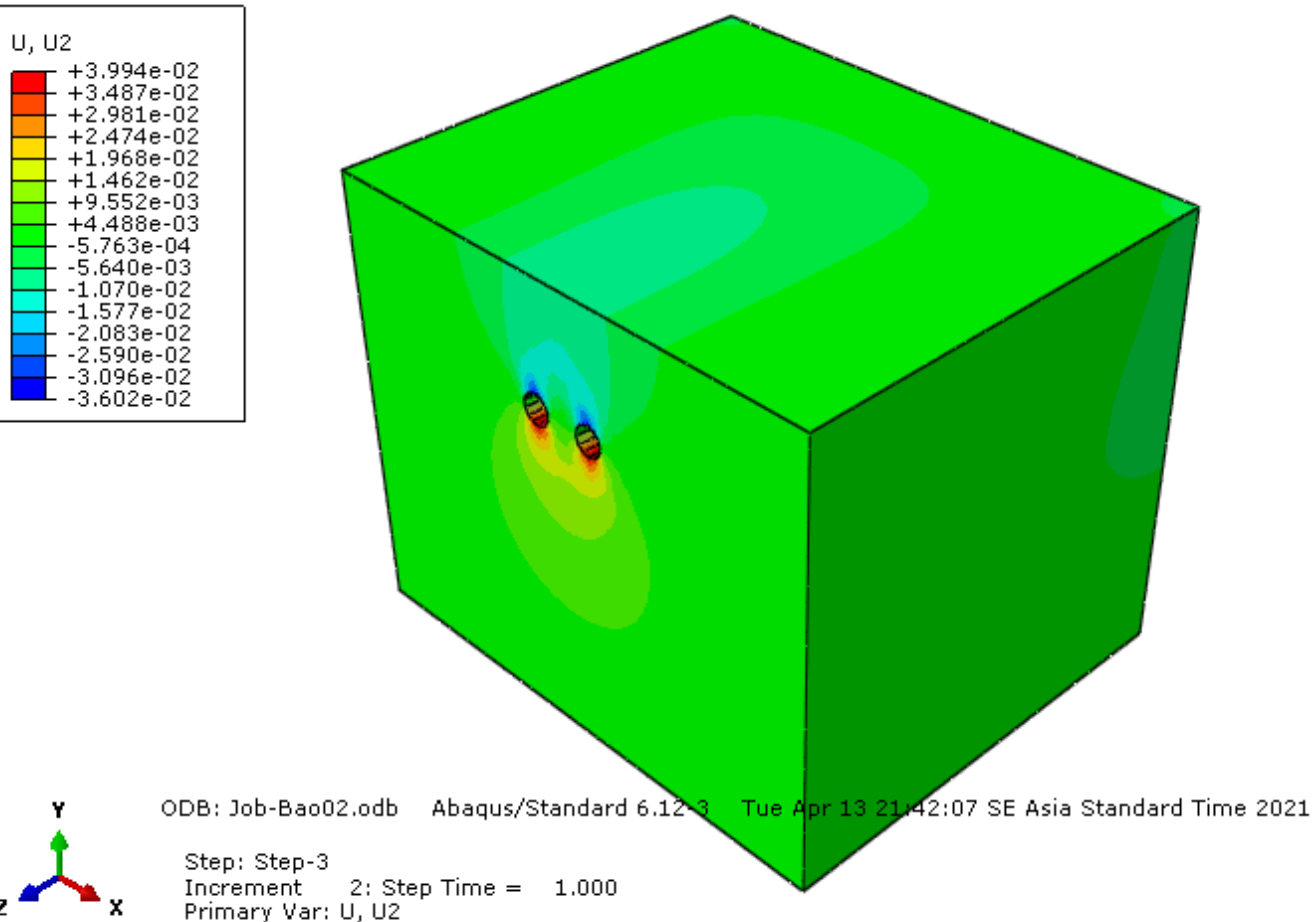

b) Sau khi thi công cả hai đường hầm.

Hình 6. Kết quả mô phỏng giá trị lún mặt đất.

Kết quả tính toán giá trị lún mặt đất lớn nhất trên mặt cắt ngang và giá trị lún mặt đất dọc trục đường hầm được thể hiện trong các Hình 7, 8.
Từ kết quả phân tích mô hình số thể hiện trên các Hình 7, 8 cho thấy: công tác thi công đường hầm gây ra lún mặt đất, sau khi thi công đường 
hầm bên trái gây ra lún mặt đất có giá trị lớn nhất là $22 \div 24 \mathrm{~mm}$ và tại vị trí gương đào đạt giá trị lún mặt đất là $7 \div 8 \mathrm{~mm}$. Sau khi thi công tiếp đường hầm bên phải tức là sau khi thi công xong hai đường hầm thì gây ra lún mặt đất có giá trị lớn nhất là $33 \div 35 \mathrm{~mm}$ và tại vị trí gương đào đạt giá trị lún mặt đất là $11 \div 12 \mathrm{~mm}$.

Khoảng cách từ mặt gương đào, $m$

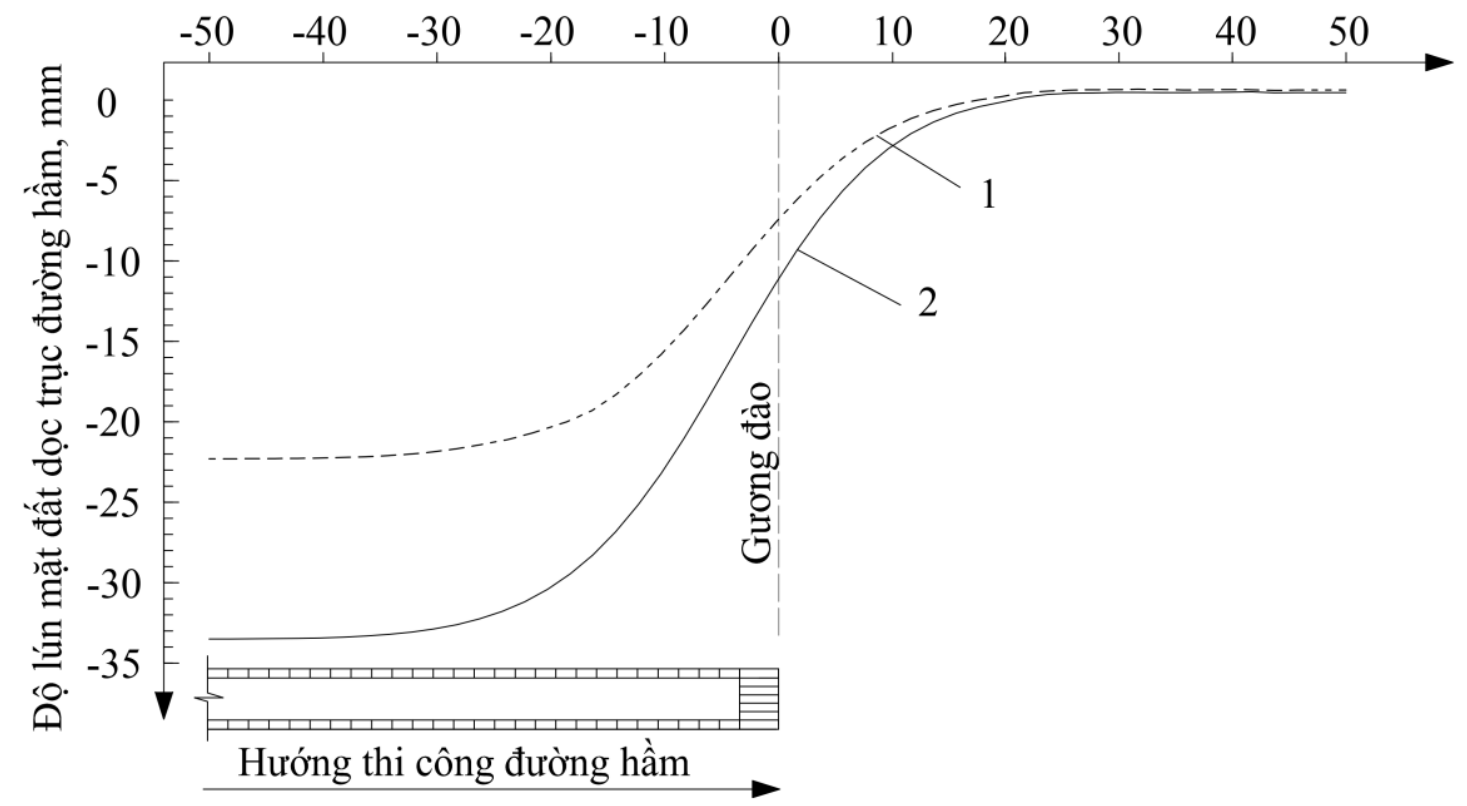

Hình 7. Đường cong lún dọc trục đường hầm: 1 - sau khi thi công đường hầm bên trái, 2 - sau khi thi công cả hai đường hầm.

Khoảng cách ngang trục đường hầm, $m$

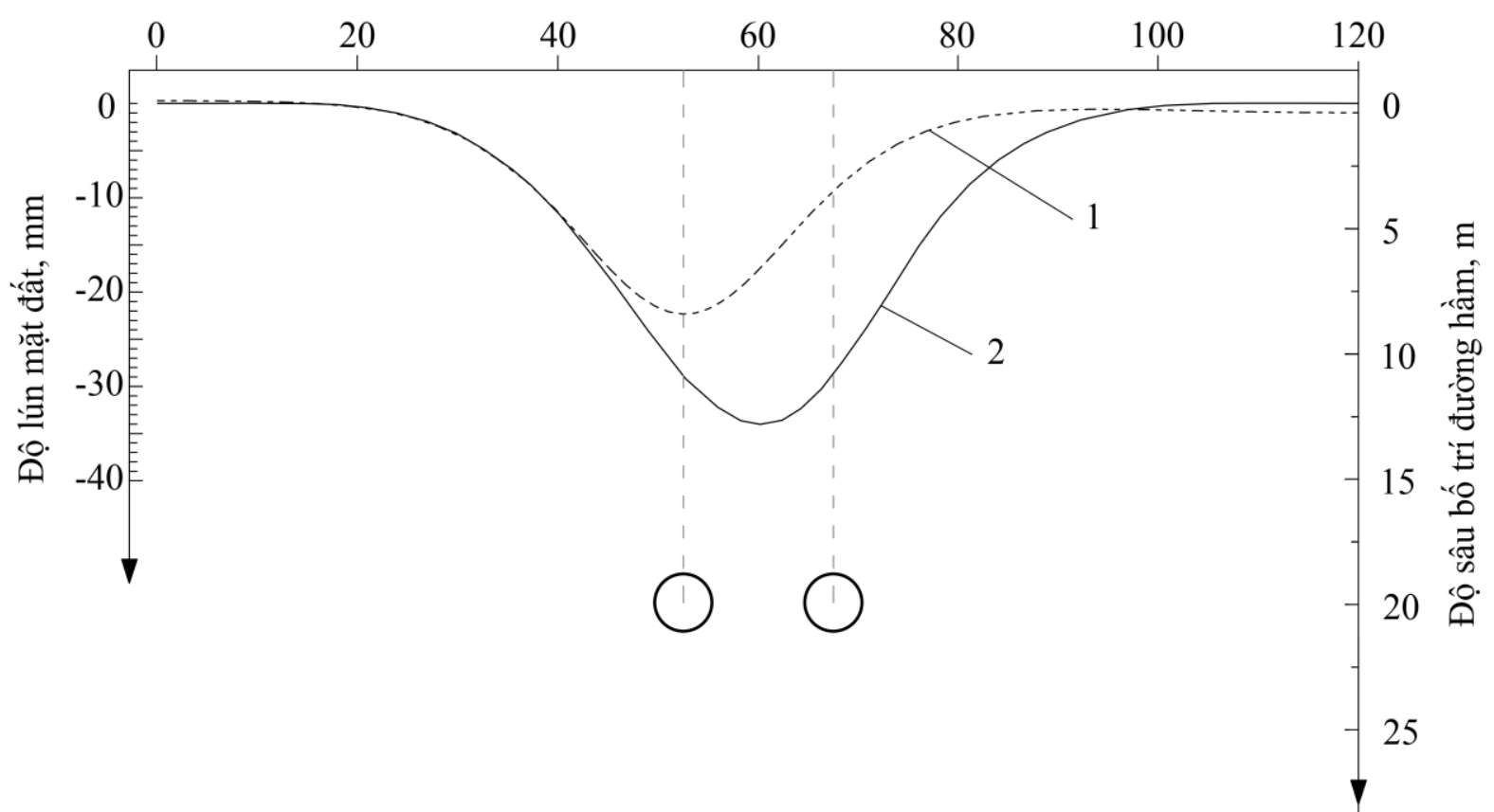

Hình 8. Đường cong lún lơn nhất theo phương ngang trục đường hầm: 1 - sau khi thi công đường hầm bên trái, 2 - sau khi thi công cả hai đường hầm. 


\section{Kết luận}

Từ kết quả phân tích mô hình số cho thấy, đối với đường hầm đô thị, cụ thể trong điều kiện tuyến Metro số 3 đoạn Nhổn - Ga Hà Nội, Thành phố Hà Nội khi thi công đường hầm dẫn đến hiện tượng dịch chuyển, lún mặt đất.

Đối với đường hầm mô phỏng tính toán trong điều kiện ở trên, tác động sau khi thi công đường hầm bên trái gây ra lún mặt đất có giá trị lớn nhất là $22 \div 24 \mathrm{~mm}$ và sau khi thi công cả hai đường hầm thì gây ra lún mặt đất có giá trị lớn nhất là $33 \div 35 \mathrm{~mm}$.

Giá trị kết quả phân tích cho thấy, giá trị lún mặt đất tại vị trí gương đào có giá trị bằng $(0,35 \div 0,40)$ lần giá trị lún mặt đất lớn nhất gây ra bởi công tác thi công đường hầm $\mathrm{S}_{\mathrm{v} \text {-guơong }}=$ $(0,35 \div 0,40) \mathrm{S}_{\mathrm{v} \text {-max }}$.

Vị trí vùng bị ảnh hưởng lún mặt đất theo chiều dọc đường hầm ở phía trước gương đào là $15 \mathrm{~m}$ và giá trị lún lớn nhất đạt được tại vị trí phía sau gương đào là $30 \mathrm{~m}$.

Vị trí vùng bị ảnh hưởng lún mặt đất theo chiều ngang có vị trí sang hai bên tới $25 \mathrm{~m}$ tính từ tim hầm.

Để giảm thiểu giá trị lún mặt đất thì công tác quy hoạch, thiết kế cần bố trí đường hầm trong khu vực có điều kiện địa chất, địa chất thủy văn ổn định, thuận lợi cho công tác thi công. Công tác thi công vận hành sử dụng máy khiên đào cần điều chỉnh giá trị áp lực lên gương đào phù hợp, không để khối đất đá sập đổ vào gương đào sẽ gây ra hiện tượng sụt lún lên đến mặt đất. Công tác phụt vữa lấp đầy khoảng trống sau vỏ chống có ý nghĩa rất lớn không chỉ tăng độ bền độ ổn định cho đường hầm mà còn giảm thiểu đáng kể giá trị lún mặt đất.

\section{Lò̀i cảm ơn}

Nhóm tác giả xin gửi lời cảm ơn đến Ban Quản lý Đường sắt đô thị Hà Nội do đã cung cấp các thông số kỹ thuật hệ thống tuyến đường sắt đô thị số 03 tại Thành phố Hà Nội.

\section{Đóng góp của các tác giả}

Tác giả Đỗ Ngọc Thái có nhiệm vụ phân tích tổng hợp và giải quyết bài toán bằng phương pháp phần tử hữu hạn, có sự đóng góp 65\%. Tác giả Nguyễn Đức Trường có nhiệm vụ thu thập số liệu đầu vào cho bài toán, kiểm ra kết quả bài toán, có sự đóng góp là 35\%.

\section{Tài liệu tham khảo}

Abaqus Inc. (2012). Abaqus User's Manual. Version 6.12. SIMULIA. 2012.773p.

Divall, S., \& Goodey, R. J. (2012). Apparatus for centrifuge modelling of twin-tunnel construction. International journal of physical modelling in geotechnics, 12(3), 102-111.

Do Ngoc Thai and Protosenya, A. G., (2017). The effect of tunnel face support pressure on ground surface settlement in urban areas due to shield tunnelling. Geo-Spatial Technologies and Earth resources(ISM -2017), 415- 420.

Design report technical design, (2012). Project: Hanoi pilot light metro line section Nhon - Hanoi railway station, package: underground section - line and stations package number: HPLMLP/CP-03 evalutation of the potential effects induced by tunnels and stations excavations in Hanoi/ project implementation consultant: systra s.a, Hanoi, Vietnam, november 2012, 45p.

Geotechnical interpretative report underground section - Design report technical design, (2012). Project: Hanoi pilot light metro line 03 Section Nhon - Hanoi Railway station. Hanoi, Vietnam. 2012, 113p.

O'Reilly, M.P. and New, B.M. (1982). Settlements above tunnels in the UK - their magnitude and prediction. Tunnelling, 82, 173-181.

Peck, R.B. (1969). Deep excavations and tunnelling in soft ground. In: Proc. 7th ICSMFE, State-of-the-art Volume, Mexico City. Mexico: Sociedad Mexicana de Mecánica de Suelos, 225-290.

Schmidt, B., (1974). Prediction of Settlements Due To Tunnelling in Soil: Three Case Histories, Proceedings, Rapid Excavation and Tunnelling Conference, V2, 1179-1199..

Protosenya, A. G. , Belyakov, N. A. , Do Ngoc Thai, (2015). The development of prediction method of earth pressure balance and earth surface settlement during tunneling with mechanized tunnel boring machines. Proceedings of the mining institute 211, 53 - 63.

Võ Trọng Hùng, Phùng Mạnh Đắc, (2005). Cơ học đá ứng dụng trong xây dựng công trình ngầm và khai 
thác mỏ. Nhà xuất bản Khoa học kỹ thuật. Hà Nội. 2005, $463 \mathrm{p}$.

Đỗ Ngọc Anh. (2016). Quy trình mới dự báo lún mặt đất phía trên hai đường hầm nằm nông trong đất mềm. Tuyển tập các công trình khoa học Kỷ niệm 50 năm thành lập Bộ môn Xây dựng công trình ngầm và mỏ, 06-2016, 32-41.

Đỗ Ngọc Thái, (2019). Phương pháp dự báo độ lún mặt đất khi xây dựng đường hầm thành phố bằng máy khiên đào. Tạp Chí Công nghiệp mỏ, số 3 năm 2019, 55-60.

Đỗ Ngọc Thái, Đặng Văn Kiên, (2019). Phân tích ổn định bề mặt gương đào khi xây dựng đường hầm trong điều kiện đất đá yếu bằng máy khiên đào. Tạp chí Khoa học Kỹ thuật Mỏ - Địa chất. 60(1), 1-6. 